\title{
ОБНАРУЖЕНИЕ СКРЫТОГО СТЕГАНОГРАФИЧЕСКОГО ВЛОЖЕНИЯ И ПРИЗНАКОВ МОНТАЖА В ОБЛАСТИ ДАННЫХ АУДИОФАЙЛА
}

\author{
(C) 2020 А. С. Гераськин ${ }^{\bowtie}$, Е. Д. Смирнов \\ Саратовский национальный исследовательский государственный университет \\ имени Н. Г. Чернышевского \\ ул. Астраханская 83, 410012 Саратов, Российская Федерация
}

\begin{abstract}
Аннотация. Наиболее часто используемые и передаваемые файлы - это аудиофайлы. Однако при такой передаче возникает вопрос об отсутствии модификации данного файла. Широкое распространение звуковых редакторов и различных программ обработки и монтажа аудиозаписей позволяют даже непрофессионалу осуществить фальсификацию фонограммы речи. Также возможна еще одна модификация аудиофайла, такая как использование его в виде стеганографического контейнера. Существует множество методов стеганографии для осуществления передачи информации под видом стандартных файлов. В связи с этим актуален вопрос по созданию методов исследования аудиозаписей и инструментальных средств анализа на наличие признаков их изменения. Поэтому в статье приводится исследование, посвященное обнаружению скрытого стеганографического вложения и признаков изменения в области данных аудиофайла с определением численных критериев принятия решения. Рассматриваются методы статистического анализа аудиофайлов на предмет внесенных изменений в области данных. При анализе внесённых изменений в аудиофайл применяют методы стегоанализа и спектральный анализ. Предлагаются алгоритмы, основанные на оценке изменения статистических характеристиках сигма-отношения «сигнал/шум», позволяющий провести оценку внесенных изменений. Описываются алгоритмы вычисления коэффициента сигма-отношения «сигнал/шум», на отдельных блоках, и на всем файле. Реализованные алгоритмы были проверены на аудиофайлах, в которые вносились следующие изменения: вставка - аудиофайл используется как стегоконтейнер; склейка - соединение двух аудиофайлов; вырезка - аудиофайл модифицируется путем удаления части информации. На основе предложенных методов проведена оценка возможных внесенных изменений. В результате эксперимента было выявлено, что с помощью методов можно оценить склейку аудиофайлов, вырезку фрагмента без предъявления оригинала. В статье приведены данные, которые показывают точность определения внесённых изменений.

Ключевые слова: стеганографическое вложение, монтаж, область данных аудиофайла, коэффициент сигма-отношения «сигнал/шум», стегоанализ аудиофайла, спектральный анализ аудиофайла, вставка в аудиофайл, склейка аудиофайла, вырезка из аудиофайла.
\end{abstract}

\section{ВВЕДЕНИЕ}

В настоящее время цифровое представление данных является наиболее часто используемым видом хранения информации. Преимуществами такого представления является возможность передачи информации на боль-

Гераськин Алексей Сергеевич e-mail: gerascinas@mail.ru шие расстояния с помощью сети Интернет. Наиболее распространёнными файлами в сети Интернет являются аудиофайлы. При получении такого файла возникает вопрос об отсутствии его модификации. Модификация могла быть сделана как третьим лицом, не участвующим в передаче и хранении информации, который намеренно пытался видоизменить файл, чтобы какие-то части данных оказались недоступны, так и сотрудником,

Контент доступен под лицензией Creative Commons Attribution 4.0 License.

The content is available under Creative Commons Attribution 4.0 License. 


\section{А. С. Гераськин, Е. Д. Смирнов}

который проявил халатность в обращении с имеющимся элементом или сделал это специально с целью скрыть часть переданной или хранящейся информации. Широкое распространение звуковых редакторов и различных программ обработки и монтажа аудиозаписей позволяют даже непрофессионалу осуществить фальсификацию фонограммы речи. В связи с этим актуален вопрос по созданию методов исследования аудиозаписей и инструментальных средств анализа на наличие признаков их монтажа [1].

Наряду с этим возможна еще одна модификация аудиофайла, такая как использование его в виде стеганографического контейнера. Существует множество методов стеганографии для осуществления передачи информации под видом стандартных файлов [2]. Аудиофайлы по сравнению с видео имеют более простую структуру и используются чаще [3].

Целью исследования является обнаружение скрытого стеганографического вложения и признаков монтажа в области данных аудиофайла с определением численных критериев принятия решения.

\section{1. МЕТОДЫ АНАЛИЗА АУДИОФАЙЛА НА НАЛИЧИЕ ВНЕСЁННЫХ ИЗМЕНЕНИЙ}

Анализ аудиофайлов на наличие внесённых изменений - это процесс, позволяющий оценить внутреннюю структуру файла на предмет обработки какой-либо программой. Необходимо сказать, что эта область развивается активно, каждый день появляются новые методы и алгоритмы для получения более точных сведений.

Для определения внесённых изменений в аудиофайл могут использоваться следующие виды анализа:

- стегоанализ;

- спектральный анализ [4].

Стегоанализ аудиофайлов заключается в нахождении факта передачи скрытой информации в имеющемся звуковом файле. Стегоанализ обычно даёт только вероятностную оценку нахождения стего в анализируемом объекте [5].

Методы, основанные на изменении значений в фазовой области, изменяют абсолютное значение фазы некоторых гармоник. Существуют разновидности, в которых восстанавливаются значения разностей фаз смежных фреймов. Делается это на основе предположения, что человеческое восприятие звука чувствительно не к абсолютному значению фазы сигнала, а к разности фаз смежных фрагментов. В других методах фаза выбранной гармоники изменяется либо на фиксированное значение, либо изменением знака действительной и мнимой частей комплексного спектра $[6,7]$.

Для детектирования секретной информации в аудиосигнале необходимо проанализировать распределение значений квадрантов фаз гармоник. В случае если во фрейм было встроено сообщение, будет заметно преобладание некоторых квадрантов [7].

Методы стегоанализа на основе методов сжатия. Обычно считается, что файл, содержащий однородные данные, имеет свою статистическую структуру. Если использовать его в качестве контейнера для секретного сообщения, то после внедрения сообщения в контейнер нарушится статистическая структура контейнера и повысится его энтропия. Таким образом, при использовании алгоритмов сжатия исходный пустой контейнер сжимается, как правило, лучше, чем заполненный. Значит, если степень сжатия предполагаемого контейнера больше некоторого порогового значения, то с большой вероятностью можно сказать, что контейнер пуст, в противном случае можно говорить о присутствии сообщения в контейнере [6].

Рассматриваемый метод заключается в сравнении коэффициентов сжатия исходного контейнера и его полностью заполненной копии. Полностью заполненная копия получается при помощи псевдослучайного изменения младших бит исходного контейнера. К обоим файлам применяется метод сжатия данных, и анализируются их коэффициенты сжатия. Если эти коэффициенты близки по 
значению, то с большой долью вероятности можно утверждать, что исходный файл содержал скрытое сообщение, иначе говорится об отсутствии секретной информации в объекте. При практической реализации этого метода контейнер и его заполненная копия делятся на несколько равных частей, что позволяет увеличить точность метода [8].

Метрика Хаусдорфа - это максимально-минимальное отношение, которое нашло множество применений в задачах сопоставления с шаблоном и восстановления на основе содержимого. Этот метод стегоанализа превосходит по скорости работы и эффективности обнаружения скрытой информации другие методы стегоанализа. Однако использование конкретного правила обнаружения скрытой информации напрямую зависит от алгоритма встраивания, что является недостатком в плане универсальности. Если алгоритм неизвестен для пользователя, то определение вставки может быть затруднено [9-11].

Стегоанализ, основанный на анализе естественных закономерностей записанной речи. Аудиосигналы обычно рассматриваются в трех основных представлениях: временном, частотно-временном и частотном. Частотно-временная форма преодолевает некоторые недостатки представления во временной или частотной области. В этом представлении аудиосигнал описывается в терминах базисных функций, которые локализованы как по времени, так и по частоте. Оконное преобразование Фурье [4] является наиболее распространенным частотно-временным разложением для аудиосигналов. Данный метод позволяет с высокой эффективностью обнаруживать вложения различными стандартными методами, в том числе и LSB. При этом рассмотренный метод не всегда сможет обнаруживать изменения в какой-то записанной музыке. Причина в том, что статистика, свойственная музыкальному файлу, возможно, будет сильно отличаться от статистики для речи. Также появляются осложнения из-за широких различий в качестве используемого файла [12].

Стегоанализ на основе обращенной психоакустической модели человеческого слуха.
Мел - психофизическая единица высоты звука, применяется главным образом в музыкальной акустике. Количественная оценка звука по высоте основана на статистической обработке большого числа данных о субъективном восприятии высоты звуковых тонов. К недостаткам использования метода на основе мел-кепстральных коэффициентов можно отнести следующее:

- плохо работает при наличии аддитивного шума (обычно используется нормализация для уменьшения влияния шума);

- мел-кепстральные коэффициенты уравнивают низкие и высокие амплитуды в спектре, что приводит к потере некоторых данных;

- может происходить удаление индивидуальных особенностей речи говорящего при нормализации данных с целью снижения искажений [13].

Как известно, в области данных аудиофайла хранится информация обо всех частотах и амплитудах. Спектральный анализ заключается в переводе аудиосигнала из временной формы в частотную для более удобного рассмотрения содержимого и определения изменений между рядом стоящими данными [14].

Пусть последовательность отсчётов $\{x(k)\}$ является периодической с периодом $N: x(k+N)=x(k)$ для любого $k$. Тогда исходный сигнал можно представить в виде суммы произведений отсчётов и смещённых во времени дельта-функций:

$$
s(t)=\sum_{k=-\infty}^{\infty} x(k) \delta(t-k T),
$$

где $x(k)-$ произвольный фрагмент длиной $k$;

$\delta(t-k T)$ - дельта-функция, которая описывается с помощью формул (2) и (3):

$$
\begin{gathered}
\delta(x)=\left\{\begin{array}{l}
\infty, x=0 \\
0, x \neq 0
\end{array},\right. \\
\int_{-\infty}^{\infty} \delta(x) d x=1 .
\end{gathered}
$$

Для представления аудиосигнала в виде зависимости частота - мощность сигнала используется набор частот от 0 до половины значения частоты дискретизации, а также мощность сигнала вычисляется по данным, 
полученным с помощью БПФ (быстрое преобразование Фурье), по следующей формуле:

$$
D_{p}=10 \lg \frac{P_{1}}{P_{0}},
$$

где $P_{0}$ и $P_{1}-$ два значения энергетической величины $P$.

Для проведения анализа аудиофайла необходимо ввести следующие статистические характеристики: среднее значение и общее сигма-отношение «сигнал/шум».

Среднее значение $\bar{x}$ - среднее арифметическое, рассчитанное путем сложения группы чисел и деления на количество этих чисел:

$$
\bar{x}=\frac{1}{n} \sum_{i=1}^{n} x_{i},
$$

где $x_{i}-i$-е число в последовательности $x$;

$n$ - количество чисел.

Общее сигма-отношение «сигнал/шум» коэффициент GSSNR, который определяется по следующим формулам:

$$
\begin{gathered}
G S S N R=\frac{\sum_{b} \sigma_{b}^{2}}{\sum_{b}\left(\sigma_{b}-\widetilde{\sigma_{b}}\right)^{2}}, \\
\sigma_{b}=\sqrt{\frac{1}{n} \sum_{\text {блок } b}\left(C_{x, y}\right)^{2}-\left(\frac{1}{n} \sum_{\text {блок } b} C_{x, y}\right)^{2}},
\end{gathered}
$$

где блок $b$ - некоторый интервал частот в спектральном представлении аудиофайла;

$\sigma_{b}$ - среднее значение в рассматриваемом блоке $b$;

$n$ - размер блока $b$;

$C_{x, y}$ - значение из блока $b$.

\section{2. АЛГОРИТМ ПОИСКА ИЗМЕНЕНИЙ В ОБЛАСТИ ДАННЫХ ФАЙЛА}

Осуществим применение приведенных выше статистических характеристик данных (6), (7) для анализа аудиофайла. Метод поиска внесённых изменений в аудиофайл заключается в анализе изменения значения коэффициента GSSNR (6) на отдельных блоках файла и на всем файле. Алгоритм вычисления коэффициента GSSNR на отдельных блоках файла определяется следующей последовательностью действий:

1. разобьем всю последовательность на блоки $b$. Изначально размер блока $b$ устанав- ливается равным 250 Гц, т.е. рассматривается набор частот от 0 до 250 Гц. Множитель, определяющий количество интервалов разбиения $k$, полагается равным 10 ;

2. если размер блока $b$ не больше чем половина значения частоты дискретизации, то к шагу к 3. Иначе выдать на выходе массив $g$, состоящий из коэффициентов GSSNR, и завершить алгоритм;

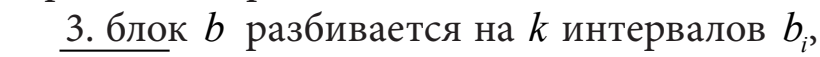
$i=\overline{0, k-1}$;

4. для каждого интервала $b_{i}$ вычисляется значение $\sigma_{b_{i}}$, используя формулу (7). В качестве $C_{x, y}$ берутся значения мощностей сигнала из рассматриваемого интервала $b_{i}, n$ полагается равным размеру интервала $b_{i}$. Также для каждого интервала вычисляется значение $\widetilde{\sigma_{b}}$, используя формулу (5);

5. используя полученные значения $\sigma_{b_{i}}$ и $\widetilde{\sigma_{b_{i}}}, i=\overline{0, k-1}$, по формуле (6) вычисляется значение коэффициента GSSNR, которое записывается в массив $g$;

6. $k=k+10$;

7. размер блока $b$ увеличивается на 250 и к шагу 2 (т.е. будут рассматриваться интервалы частот от 0 до 250 Гц, от 0 до 500 Гц и т.д. до конца файла).

Алгоритм вычисления значения коэффициента GSSNR по всему аудиофайлу может быть представлен следующей последовательностью действий:

1. разобьем всю последовательность на блоки $b$. Размер блока $b$ устанавливается фиксированным значением 250 Гц;

2. пока не достигнута половина значения частоты дискретизации выполняем действия 3 и 4 ;

3. для рассматриваемой последовательности вычисляется значения $\sigma_{b}$ и $\widetilde{\sigma_{b}}$, используя формулы (7) и (5);

4. блок $b$ сдвигается вправо на 250 Гц;

5. вычисляется коэффициент GSSNR для всего аудиофайла по формуле (6).

\section{3. РЕЗУЛЬТАТЫ ИССЛЕДОВАНИЙ И ИХ ОБСУЖДЕНИЕ}

Результаты работы алгоритмов представлена на рис. 1. Красной линией отображается 
значение GSSNR для всего файла, зелёными точками значения массива $g$, соединённые между собой синим пунктиром. График, представленный на рис. 1, представляет собой нормальное поведение кривой, имеющей максимум в области между низкими и средними частотами и стремящейся к коэффициенту GSSNR по всему аудиофайлу.

B ходе экспериментальных исследований была подготовлена серия звуковых файлов формата WAVE. Использовались файлы формата РСM, частота дискретизации 44 кГц, количество каналов - 2. Представленные алгоритмы были протестированы на 80 аудиофайлах, в которые вносились следующие изменения:

- вставка - аудиофайл использовался в качестве контейнера для внедрения скрытого сообщения с помощью метода наименьших значащих бит (LSB) с последовательным изменением бит с начала файла. Использовалась различная степень наполнения: $10 \%$, $20 \%, \ldots, 90 \%$;

- склейка - соединение двух (или нескольких) аудиофайлов [15]. Использовалось различное процентное отношение длин вырезаемых фрагментов к длине всего файла: 10 $\%, 20 \%, \ldots, 50 \%$;

- вырезка - аудиофайл модифицируется путем удаления части информации. Использовалось различное процентное отношение длин склеиваемых фрагментов: $10 \%, 20 \%, \ldots$, $50 \%$.

Следует отметить, что проводимые модификации аудиофайла на слух неразличимы.

Если файл был изменен, например, было проведено склеивание различных фрагментов одного и того же (или разных) файла на слух неотличимых друг от друга, то получается следующий график зависимости коэффициента GSSNR от частоты. Измененный график представлен на рис. 2.

Следующим примером является аудиофайл, который был модифицирован путем вставки. В качестве примера вставки берётся аудиофайл с секретным сообщением, которое добавляется к исходному файлу с использованием метода наименьшего значащего бита (LSB). Как показало исследование, обнару-

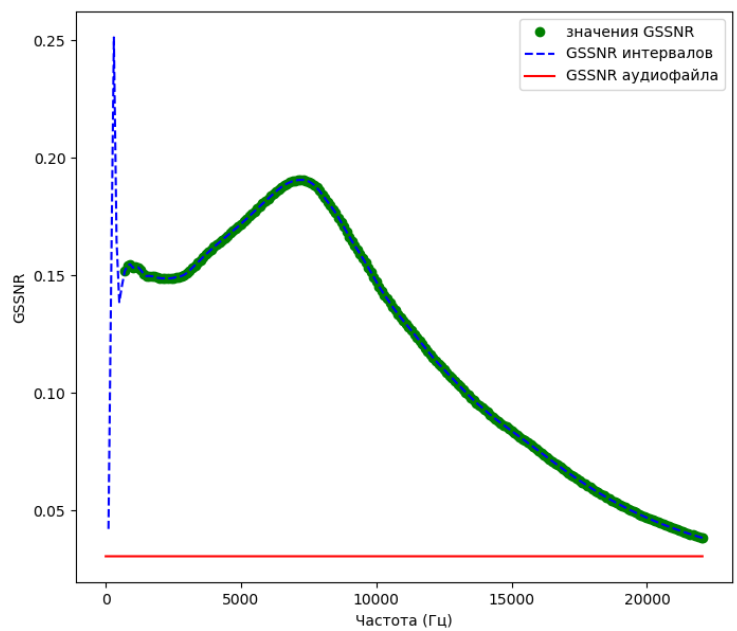

Puс. 1. Зависимость коэффициента GSSNR от частоты для «чистого» аудиофайла

[Fig. 1. Dependence of the GSSNR on the frequency for a "clean" audio file]

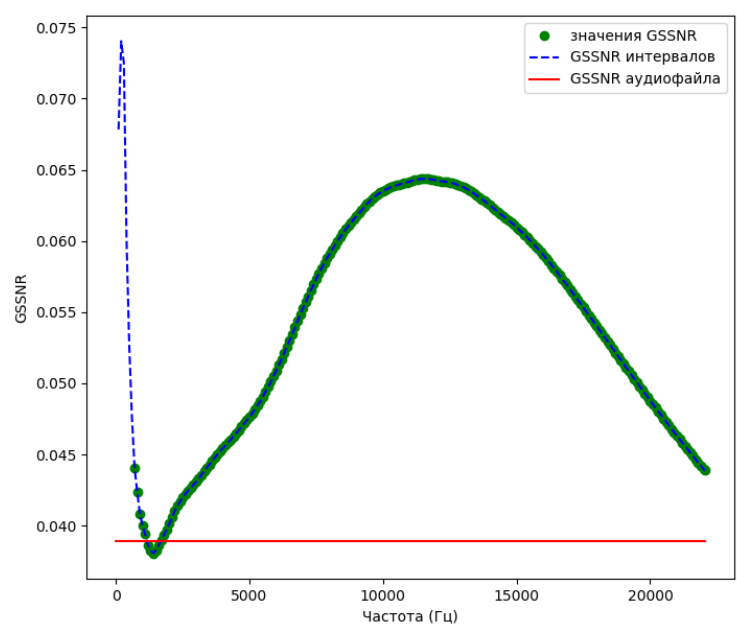

Puс. 2. Зависимость коэффициента GSSNR от частоты для склеенного аудиофайла

[Fig. 2. Dependence of the GSSNR on the frequency for a merged audio file]

жение изменений в аудиофайле с помощью предложенного метода возможно, если степень наполнения контейнера менее 50 \%. На рис. 3 приведен пример файла, заполненного на $10 \%$. Как видно из графика, так же, как и в случае склейки на рис. 2, получается график, который отличается от исходного, представленного на рис. 1. Поэтому можно сделать вывод о том, что в аудиофайл вносились изменения.

Однако если контейнер заполнить на 70 \%, как показано на рис. 4, то график становится более гладким и не имеет явных признаков изменения. На графике не наблюдается точек, 


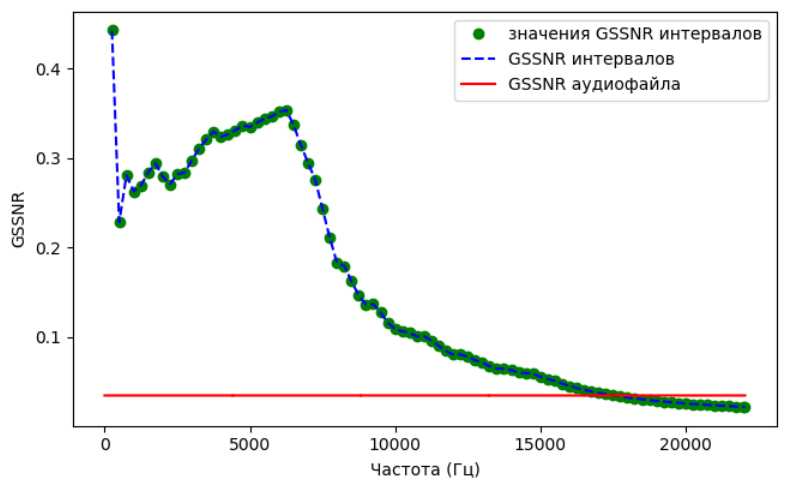

Puc. 3. Зависимость коэффиичента GSSNR от частоты для аудиобайла, в который производилась вставка, контейнер заполнен на $10 \%$

[Fig. 3. Dependence of the GSSNR on the frequency for an audio file with an embedded message (the container is $10 \%$ filled)]

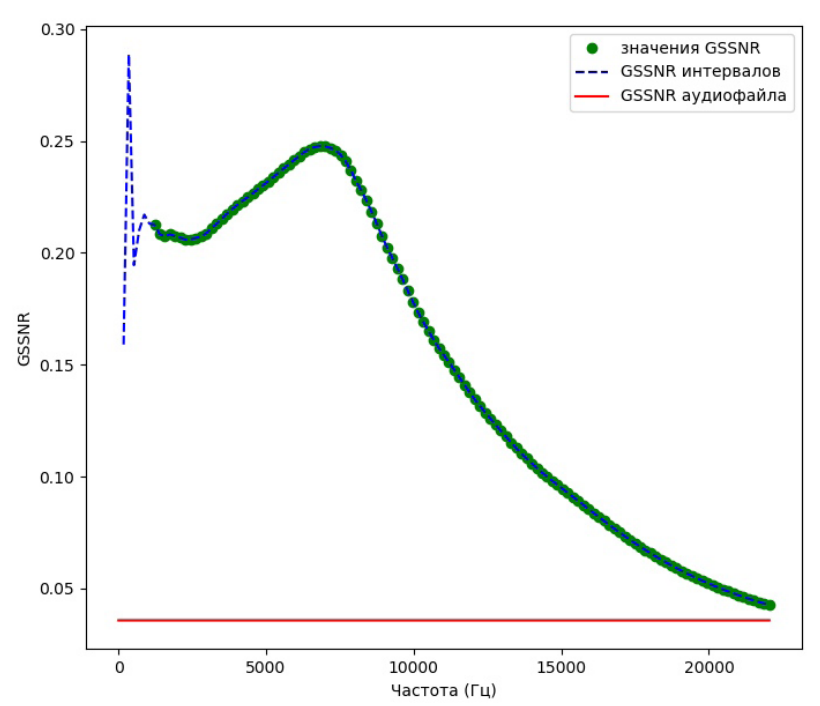

Puc. 4. Зависимость коэффициента GSSNR от частоты для аудиофайла, в который производилась вставка, контейнер заполнен на $70 \%$

[Fig. 4. Dependence of the GSSNR on the frequency for an audio file with an embedded message (the container is $70 \%$ filled)]

выходящих за среднее значение коэффициента GSSNR.

Рассмотрим пример с вырезкой фрагмента из файла. Как видно из графика, представленного на рис. 5, так же, как и в случае склейки, получается изображение в виде параболы, что говорит о том, что файл был изменён.

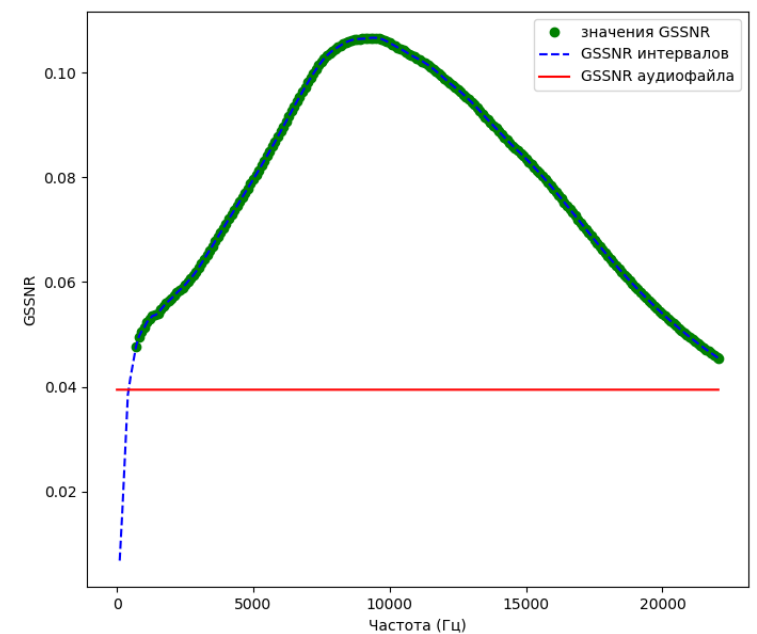

Puс. 5. Зависимость коэфбиииент GSSNR от частоты для аудиобайла с вырезкой

[Fig. 5. Dependence of the GSSNR on the frequency for a clipped audio file]

Для определения вносимых изменений используется правило того, как ведёт себя зависимость коэффициента GSSNR от частоты. Для коэффициента GSSNR зависимость должна представлять собой функцию, которая сначала имеет минимум в области между низкими и средними частотами (от 2500 до 4000 Гц) при этом не достигает значения коэффициента GSSNR по всему файлу, потом возрастает, имеет максимум в диапазоне средних частот (до 7500 Гц), а потом стремится к значению коэффициента GSSNR по всему файлу как показано на рис. 1. Для типов аудиофайлов «Склейка» наблюдается минимум ниже значения коэффициента GSSNR по всему файлу в диапазоне до 5000 Гц и смещение максимума в область от 10000 до 15000 Гц, как показано на рис. 2. Для типов аудиофайлов «Вырезка», представленного на рис. 5, минимум не наблюдается, а максимум смещен в область от 80000 до 12000 Гц.

Точность определения внесённых изменений для типов аудиофайлов «Склейка» и «Вырезка» составляет $90 \%$ и 95\% соответственно. Для типов аудиофайлов «Вставка» методы дает 50 \% правильных определений. В результате тестирования для типов аудиофайлов «Вставка» было замечено, что чем выше наполненность контейнера, тем ниже определение аудиофайла как «с изменениями», поскольку он становится более однородным. 
Для типов аудиофайлов «Склейка» и «Вырезка» существует небольшая погрешность, связанная с особенностями предложенного алгоритма. Некоторые изменения попадали на стык двух блоков $b_{1}$ и $b_{2}$, в результате чего они попадали не в один, а в разные блоки, поэтому резкого возрастания коэффициента GSSNR в блоке не происходило.

\section{ЗАКЛЮЧЕНИЕ}

Предложены статистические методы анализа аудиофайла на предмет внесенных изменений. C помощью данных методов можно оценить склейку аудиофайлов, вырезку фрагмента без предъявления оригинала. На основании результатов, полученных из экспериментальных исследований, было выявлено, что точность определения внесённых изменений для типов аудиофайлов «Склейка» и «Вырезка» составляет $90 \%$ и $95 \%$ соответственно. Для типов аудиофайлов «Вставка» методы дают 50 \% правильных определений.

\section{КОНФЛИКТ ИНТЕРЕСОВ}

Авторы декларируют отсутствие явных и потенциальных конфликтов интересов, связанных с публикацией настоящей статьи.

\section{СПИСОК ЛИТЕРАТУРЫ}

1. Горшков, Ю. Г. Экспертиза подлинности аудиозаписей, основанная на новой технологии многоуровневого вейвлет-анализа / Ю. Г. Горшков // Безопасные информационные технологии : сб. тр. Восьмой всероссийской научно-технической конференции. НУК «Информатика и системы управления». Под. ред. М. А. Басараба (Москва, 06-07 декабря 2017 г.). Москва - 2017. - С. 131-134.

2. Конахович, Г. Ф. Компьютерная стеганография. Теория и практика / Г. Ф. Конахович, А. Ю. Пузыренко. - Киев : МК-Пресс, 2006. $286 \mathrm{c}$.

3. Некоторые аспекты защиты информации системы обработки мультимедийных данных / Р. Д. Апсалямова [и др.] // Современ- ные наукоемкие технологии. - 2016. - № 10 (часть 1). - С. 24-28.

4. Афонский, А. А. Цифровые анализаторы спектра, сигналов и логики / А. А. Афонский, В. П. Дьяконов. - Москва : Солон-Пресс, 2009. - 248 c.

5. Тu, А. Е. Исследование вносимых искажений методами стеганографии в аудиофайлы различных форматов / А. Е. Ти, И. А. Кривошеев // Информационные технологии XXI века : сб. науч. тр. отв. ред. В.В. Воронин. - Хабаровск : Изд-во Тихоокеан. гос. ун-та, 2018. С. 67-74.

6. Есин, М. К. Метод стегоанализа аудиофайлов, базирующийся на алгоритмах сжатия / М. К. Есин // Экспериментальные и теоретические исследования в современной науке : сб. тp. XL международной научно-практической конференции (Новосибирск, 29 мая 2019 г.). Новосибирск - 2019. - С. 5-11.

7. Кокорин, П. П. О методах стегоанализа в аудиофайлах / П. П. Кокорин // Труды СПИИPAH. - 2007. - № 4. - С. 239-246.

8. Очимов, С. Ю. Стегоанализ аудиофайлов, базирующийся на алгоритмах сжатия / С. Ю. Очимов // Журнал Вестник СибГУТИ. 2010. - № 1. - С. 33-40.

9. Ghasemzadeh, H. Comprehensive review of audio steganalysis methods / H. Ghasemzadeh, M. H. Kayvanrad // IET Signal Processing. - 2018. - V. 12. - P. 673-687, doi: 10.1049/ iet-spr.2016.0651.

10. Geetha, S. Audio steganalysis with Hausdorff distance higher order statistics using a rule based decision tree paradigm / S. Geetha, N. Ishwarya, N. Kamaraj // Expert Systems with Applications. - 2010. - V. 37. - P. 7469-7482, doi: 10.1016/j.eswa.2010.04.012.

11. Liu, Y. A novel audio steganalysis based on high-order statistics of a distortion measure with Hausdorff distance / Y. Liu, K. Chiang, C. Corbett, R. Archibald, B. Mukherjee, D. Ghosal // Springer. - 2008. - V. 5222. - P. 487-501, doi:10.1007/978-3-540-85886-7_33

12. Johnson, M. K. Steganalysis of recorded speech / M. K. Johnson, S. Lyu, H. Farid // Proc. SPIE. - 2005. - V. 5681. - P. 664-672, doi:10.1117/12.586941. 


\title{
А. С. Гераськин, Е. Д. Смирнов
}

13. Ghasemzadeh, H. Audio steganalysis based on reversed psychoacoustic model of human hearing / H. Ghasemzadeh, M. T. Khass, M. K. Arjmandi // Digital signal processing. 2016. - V.51. - P.133-141, doi:10.1016/j. dsp.2015.12.015.

14. Стивен, С. Цифровая обработка сигналов. Практическое руководство для инженеров и научных работников / С. Стивен; пер. с англ. А. Ю. Линовича, С. В. Витязева, И. С. Гусинского. - М. : ДМК-Пресс, 2018. - 718 с.
15. Ильяшенко, И. Д. Анализ признаков из wavenet автоэнкодера в задаче обнаружения искусственных искажений в аудиофайлах / И. Д. Ильяшенко, Р. С. Насретдинов // Проблемы правовой и технической защиты информации. - 2018. - № 6 - С. 39-46.

Гераськин Алексей Сергеевич - канд. пед. наук, доцент кафедры теоретических основ компьютерной безопасности и криптографии, Саратовский национальный исследовательский государственный университет имени Н. Г. Чернышевского.

E-mail: gerascinas@mail.ru

ORCID: https://orcid.org/0000-0002-3118-1022

Смирнов Егор Дмитриевич - студент кафедры теоретических основ компьютерной безопасности и криптографии, Саратовский национальный исследовательский государственный университет имени Н. Г. Чернышевского.

E-mail: smirnoved5@mail.ru

ORCID: https://orcid.org/0000-0002-1304-8084

DOI: https://doi.org/10.17308/sait.2020.2/2917

ISSN 1995-5499

Received 08.05.2020

Accepted 15.06.2020

\section{DETECTING AUDIO STEGANOGRAPHY AND SIGNS OF MODIFICATION IN THE DATA OF AUDIO FILES}

\author{
(c) 2020 A. S. Geraskin ${ }^{\bowtie}$, E. D. Smirnov \\ Saratov State University \\ 83, Astrakhanskaya Street, 410012 Saratov, Russian Federation
}

\begin{abstract}
Annotation. The most commonly used and transferred files are audio files. However, when transferring such files, we face the problem of detecting any modifications. The widespread use of sound editors and various programmes for the processing and editing of audio files makes it possible for anyone to falsify speech phonograms. Another type of modification involves using audio files as steganographic containers. There are a large number of steganography techniques for the covert transmission of information within standard files. In this regard, it is important to develop analytical tools and methods for the detection of modifications in audio files. Therefore, the article presents a study on the detection of hidden steganographic messages and signs of modifications in the data area of audio files based on determining the numerical criteria for decision making. The article considers methods for the statistical analysis of audio files for any modifications in the data. When analysing the alterations made to audio files, steganalysis methods and spectral analysis were used. The article presents algorithms based on the assessment of changes in the statistical characteristics of the signal-to-noise ratio, which make it possible to
\end{abstract}

Geraskin Aleksey S.

e-mail: gerascinas@mail.ru 
evaluate the modifications. Algorithms for calculating the coefficient of the signal-to-noise ratio are described both for separate blocks and for the whole file. The implemented algorithms were tested on audio files, which were preliminary modified in the following ways: embedding - using the audio file as a stegocontainer; merging - using two audio files to make one; clipping modifying the audio file by deleting some of the information. Using the proposed methods, we evaluated the possibility of modifications made to the files. The experiment demonstrated that the presented methods allow the detection of merged audio files and removed fragments without using the original. The article provides data that shows the accuracy of modification detection.

Keywords: steganographic attachments, editing, audio file data, sigma-to-noise ratio, steganalysis of audio files, spectral analysis of audio files, embedding, audio files merging, audio clipping.

\section{CONFLICT OF INTEREST}

The authors declare the absence of obvious and potential conflicts of interest related to the publication of this article.

\section{REFERENCES}

1. Afonskij A. A. and D’jakonov V. P. Cifrovye analizatory spektra, signalov i logiki [Digital analyzers of the spectrum, signals and logic]. 2009. Moscow, Solon-Press. (in Russian)

2. Apsalyamova R. D., Dolmatova Ya. G, Dushkin A. V., Panychev S. N. and Sakharov S. L. Some aspects of the protection of multimedia information processing system. Modern high technologies. 2016. (10). P. 24-28. (in Russian)

3. Esin M. K. Audio file steganalysis method based on compression algorithms. Experimental and theoretical studies in modern science: collection of books. readings: XL International Scientific and Practical Conference, 29 May 2019, Novosibirsk. 2019. P. 5-11. (in Russian)

4. Geetha S., Ishwarya N. and Kamaraj N. Audio steganalysis with Hausdorff distance higher order statistics using a rule based decision tree paradigm. Expert Systems with Applica-tions. 2010. 37. P. 7469-7482. Available at: doi: 10.1016/j.eswa.2010.04.012.

5. Ghasemzadeh H., Khass, M. T. and Arjmandi M. K. Audio steganalysis based on reversed psychoacoustic model of human hearing. Digital signal processing. 2015. 51. P. 133-141. Available at: doi:10.1016/j.dsp.2015.12.015.

6. Ghasemzadeh H. and Kayvanrad M. H. Comprehensive review of audio steganalysis methods. IET Signal Processing. 2018. 12. P. 673687. Available at: doi: 10.1049/iet-spr.2016.0651.
7. Gorshkov Ju. G. Jekspertiza podlinnosti audiozapisej, osnovannaja na novoj tehnologii mnogourovnevogo vejvlet-analiza [Examination of the authenticity of audio recordings based on the new technology of multilevel wavelet analysis]. In: Basaraba, M.A. Bezopasnye informacionnye tehnologii readings: The Eighth All-Russian Scientific and Technical Conference, 06-07 December 2017, Moscow, Russia. NUK «Informatika i sistemy upravlenija», 2017. P. 131-134. (in Russian)

8. Il'jashenko I. D. and Nasretdinov R. S. Analiz priznakov iz wavenet avtojenkodera $\mathrm{v}$ zadache obnaruzhenija iskusstvennyh iskazhenij $\mathrm{v}$ audiofajlah [Analysis of signs from wavenet autoencoder in the problem of detecting artificial distortions in audio files]. Problems of legal and technical protection of information. 2018. (6). P. 39-46. (in Russian)

9. Johnson M. K., Lyu S. and Farid H. Steganalysis of recorded speech. Proc. SPIE. 2005. 5681. P. 664-672. Available at: doi:10.1117/12.586941

10. Kokorin P. P. About methods of stegoanalysis in audio files. SPIIRAS Proceedings. 2007. (4). P. 239-246. (in Russian)

11. Konakhovich G. F. and Puzyrenko A. Ju. Komp'juternaja steganografija. Teorija i praktika [Computer steganography. Theory and practice]. Kiev: MK-Press, 2006 (in Russian)

12. Liu Y., Chiang K., Corbett C., Archibald R., Mukherjee B. and Ghosal D. A novel audio steganalysis based on high-order statistics of a distortion measure with Hausdorff distance. Springer. 2008. 5222. P. 487-501. Available at: doi:10.1007/978-3-540-85886-7_33

13. Ochimov S. Yu. Steganalysis of audiodata based on data compression algorithms. Vestnik SibGUTI. 2010. (1). P. 33-40. (in Russian) 
14. Steven W. Smith Digital signal processing Apractical guide for engineers and scientists Translated from English by Linovicha A. Ju., Vitjazeva S. V. and Gusinskogo I. S. Moscow : DMKPress, 2018 . (in Russian)

15. Ti A. E. and Krivosheev I. A. Issledovanie vnosimyh iskazhenij metodami steganografii $\mathrm{v}$ audiofajly razlichnyh formatov [Investigation of introduced distortion by steganography methods in audio files of various formats]. Informacionnye tehnologii XXI veka, Izd-vo Tihookean the Pacific. state University, 2018. P. 67-74. (in Russian)

Geraskin Aleksey S. - PhD in Pedagogics, Associate Professor, Department of Theory of Computer Security and Cryptography, Saratov State University.

E-mail: gerascinas@mail.ru

ORCID: https://orcid.org/0000-0002-3118-1022

Smirnov Egor D. - student, Department of Theory of Computer Security and Cryptography, Saratov State University.

Email: smirnoved5@mail.ru

ORCID: https://orcid.org/0000-0002-1304-8084 